\title{
Chronic recurrent multifocal osteomyelitis in a 9 year old girl: A Case Report
}

\author{
Samaj Gautam 1*, Nabees Man Pradhan², Bidur Gyawali \\ National Academy of Medical Sciences, Kathmandu, Nepal
}

${ }^{1}$ Consultant Orthopaedic Surgeon, Department of orthopedics, NAMS

${ }^{2}$ Professor, Department of orthopedics, NAMS,

${ }^{3}$ Consultant Orthopaedic Surgeon, Department of orthopedics NAMS

\begin{abstract}
Background and Objectives: Chronic recurrent mutlifocal osteomyelitis (CRMO) is an extremely rare skeletal disorder in the younger population. It presents with multifocal bony lesions that often mimic more sinister diagnoses such as neoplasm. The cause of this condition remains unknown and there is limited evidence on effective treatment.

Presentation of Case: A 9-year-old girl presented to our institution with non-traumatic onset of left leg pain. After failed conservative management, radiographs and MRI were obtained exhibiting a bony lesion of the proximal tibia resembling osteomyelitis. The patient was nonresponsive to antibiotics, so corticotomy and drainage was done in which only blood came out of the lesion, no pus was seen. Patient improved dramatically but again she developed similar symptoms and signs on right leg. Biopsy from left leg suggested no significant findings.

Discussion: Chronic recurrent multifocal osteomyelitis presents in patients with periodic fevers, bone pain and bone lesions that can develop anywhere in the body. This is a rare disease, which has been found to affect more girls than boys.

Conclusion: CRMO should be considered as a differential diagnosis for chronic bone pain with affinity for the long bones of the lower extremity in children and adolescents.

Key words: Chronic recurrent multifocal osteomyelits (CRMO), Corticotomy
\end{abstract}

\section{INTRODUCTION}

Chronic Recurrent Multifocal Osteomyelitis is an auto-inflammatory disease that mostly affects children. The causes of the disease have been linked to gene mutations in some cases, especially on chromosome18q in one family. More research is needed on the genetic causes of this challenging condition [1].

CRMO presents in patients with periodic fevers, bone pain and bone lesions that can develop anywhere in the body. This is a rare disease, which has been found to affect more girls than boys. The peak for symptom 
incidence is around 10 years of age, but symptoms can be present from toddler or preschool age to even when patients are on middle age or older. Some patients can have many affected areas in their body, or only a few sites in their body. Children will develop deep aching pain, limping, tenderness over affected areas and often fevers. Common sites are the long bones of the body, clavicle and shoulder girdle. The spine, ankles and feet may also be affected.

\section{CASE REPORT}

A 9 yrs/Girl from Melung, Dolkha presented to OPD on $26^{\text {th }}$ May 2014 with a chief complain of pain over the left leg and limping of left leg for 7 days .Pain was excruciating in nature, continuos throughout the day, more severe in night time. Pain used to be relieved by analgesics but for the short period only. Pain was associated with limping of the left leg (unable to bear weight on the affected limb). There was no history of any trauma, fever or any constitutional symptoms. On examination general condition was fair, normal nutritional status. Vital were stable. On local examination of left leg the overlying skin was normal, no ovoius swelling or redness with no increase in temperature. Mild tenderness was noted.

On routine blood investigation total leucocyte count was $9100 / \mathrm{cmm}$, with neutrophils 55\% and lymphocytes 48\%. Haematocrit was 37, with ESR $25 \mathrm{~mm}$ in first hour and CRP $21 \mathrm{mg} \%$. On plain X-ray ( figure 1) and USG of the left leg no abnormality noted. All findings were not suggestive of any specific disease, so we ordered MRI of left leg which showed features suggestive of acute osteomyelitis in tibial diaphysis with minimal collection in subperiosteum and surrounding periosteum (figure 2). So we made a diagnosis of Acute
Osteomyelitis of tibia and did emergency corticotomy and drainage. But for our surprise from the lesion no pus came out only blood oozed out, Patient improved markedly, Pain subsided dramatically.

But after three days patient developed pain in the right leg with similar nature and signs. Similarly the routine blood investigation, Xray (figure 2) and USG were insignificant. MRI of right leg showed similar features as of left leg. (figure 2 ). Now we had thought of a different diagnosis. On detailed past history the patient told that she used to have similar kind of symptoms on and off earlier too for one year .The character of pain was similar but with mild in intensity which used to relieve by short course of analgesics. Diagnosis of Chronic Recurrent Multifocal Osteomyelitis was made. Only supportive treatment with fluids and analgesics was continued. The patient improved in a week. Eagerly awaited biopsy report came which showed only few inflammatory cells with lymphocytes predominant which supported our diagnosis.

\section{DISCUSSION}

Chronic recurrent multifocal osteomyelitis (CRMO) ("multifocal" because it can occur in different sites, primarily bones; "osteomyelitis" because it is very similar to that disease but without any infection). It is also known as chronic recurring multifocal osteomyelitis, a rare condition in which the bones have lesions, inflammation, and pain. Its definition is evolving. Many articles highlighted CRMO as an autoimmune disease that has symptoms similar to osteomyelitis, but without the infection. Recent research now classifies CRMO as an inherited autoinflammatory disease but have yet to isolate the exact gene responsible for it. 


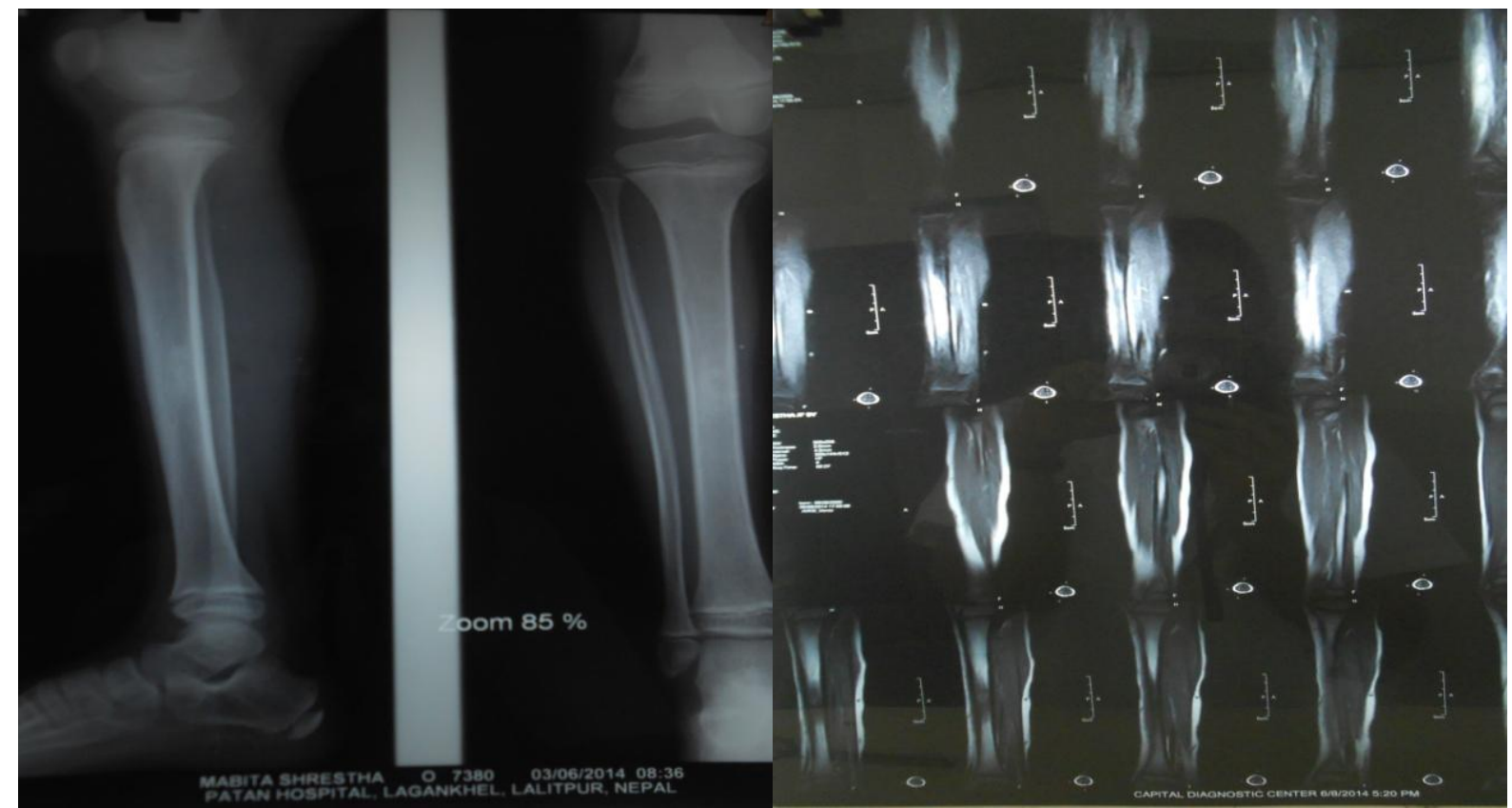

Figure 1: X-ray and MRI of left leg.

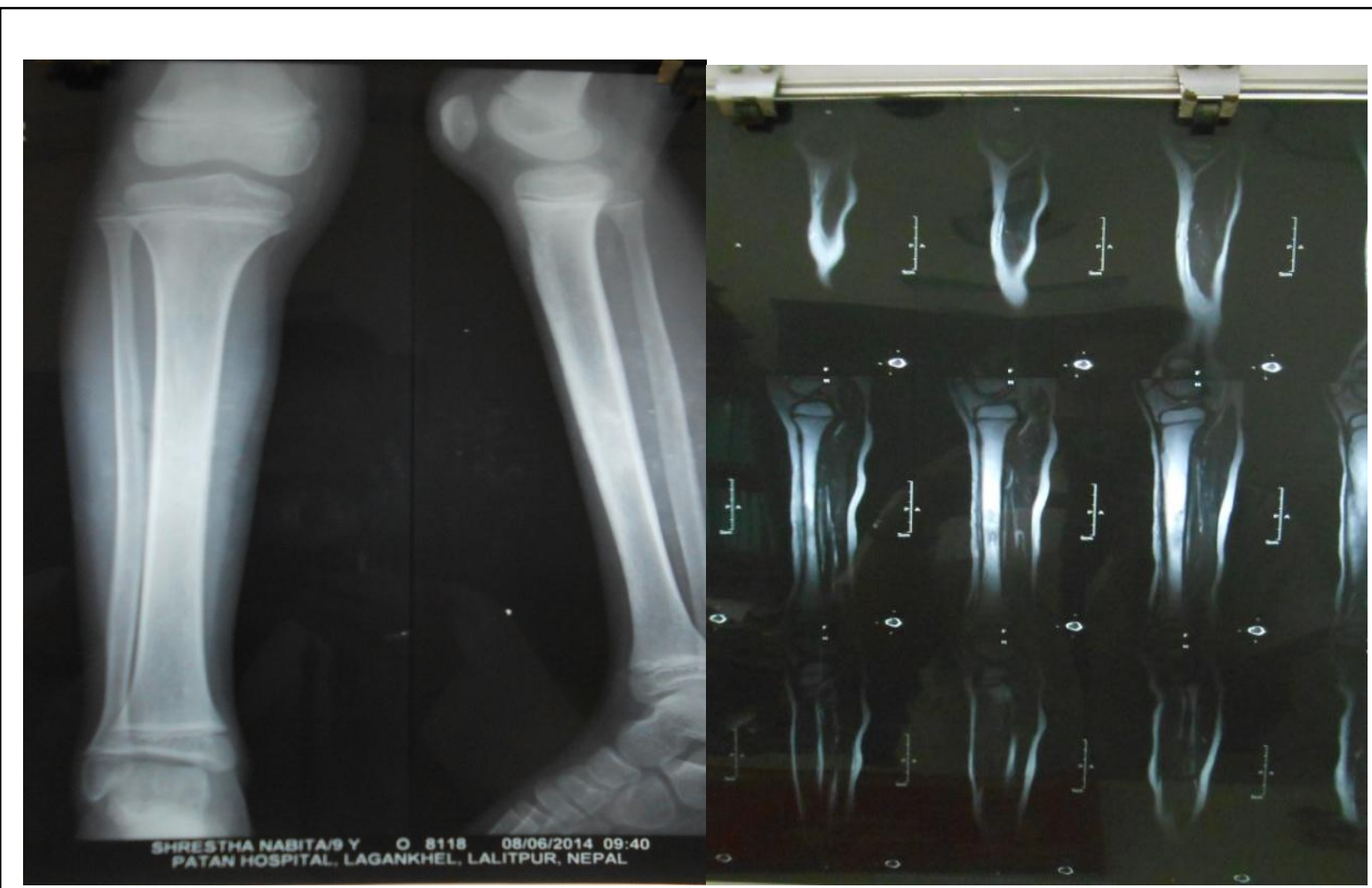

Figure 2: X-ray and MRI of right leg.

The new professional theories seem to move towards the direction of an inherited gene [1].

Patients may also present with skin manifestations, such as psoriasis, acne, pustules on the hands and feet, (SAPHO) syndrome. Some patients with CRMO also have uveitis or inflammatory bowel disease. Majeed, another autoinflammatory disease, 
involves CRMO, along with features of congenital dyserythropoietic anemia and Sweet Syndrome [2]. Majeed is a very rare disease, linked to an autorecessive inheritance of an LPIN2 gene mutation from both parents [3].

Mostly children are diagnosed with CRMO (median age of 10) but adults though rare do get it. CRMO occurs in 1: 1,000,000 and primarily in girls with a $5: 1$ ratio. Inflammation and/or lesions are best seen in MRI or bone scan [4]. However, C-reactive protein level, erythrocyte sedimentation rate, peripheral leucocytes level, ferritin level, antinuclear antibodies level and rheumatoid factor status are few laboratory test which help to discover inflammation [5].

As per case report published in journal of Canadian Chiropractic Association in Dec 2013, a 13 year old athlete girl had CRMO with no ovious cause and significant findings. Similarly another case reported in Nov 2014 had bilateral acute osteomyelitis of tibial diaphysis with no underlying cause. An extended report by $\mathrm{H}$ J Girlschick published in EULAR journal of Rheumatic disease followed 30 cases of CRMO for 5.6 years and found that the histology of the affected site showed less inflammatory cells.

CRMO patients suffer from inflammation and possibly intense pain. As such, the most common prescription is for antiinflammatory such as NSAIDs and steroids. The goal of treatment is to reduce the body of inflammation and that should ameliorate CRMO. Antibiotics are not commonly prescribed because there is no bacterial or fungal infection. But some do prescribe Azithromycin because; in addition to its antibacterial properties it also has anti- inflammatory and immune-modulatory effects.

Schilling and Wagner wrote an article that CRMO patients seem to improve greatly with Azithromycin. In one study, 7 out of 13 patients, mainly teenager, showed a fast clinical improvement after they were started on Azithromycin. The immediate therapeutic effect of Azithromycin in patients with CRMO was surprising and lead to the hypothesis that Azithromycin could have an antiphlogistic in addition to its antibiotic effect. Some have done study using interferon Gamma in the treatment of chronic Recurrent Multifocal osteomyelitis [6].

A Malaysian clinic had a similar approach. A 9 yr old girl underwent curettage through a small corticotomy on the first metatarsal bone. The pain and swelling improved dramatically and the patient was able to walk without pain in two weeks time. Since CRMO is an auto-inflammatory condition it can wax and wane. Flare ups are common with periods of remission in between. The prognosis can vary among individuals, some children may go into complete remission when they become adults and some may continue with this disease.

\section{CONCLUSION}

A doctor could easily misdiagnose CRMO as muscle spasms or simple inflammation and routinely prescribe anti-inflammatory medicines, which is the normal treatment for CRMO. Many childhood aches and pains are dismissed as growing pains however the pain of CRMO is more painful and complex than this. CRMO has deep, aching pain, swelling, and a possible fever but not always. Without an x-ray, MRI, or bone scan, the bone lesions will go undetected [4]. A diagnosis of CRMO is often made after a bone biopsy and MRI have 
ruled out other diseases that causes painful bone lesions/tumors such as Osteomylitis, Leukemia, Lymphoma, Ewine Sarcoma, Rhabdomyosarcoma, Neuroblastoma metastasis, Eosinophilic Granuloma, or Lanerhans cell histiocytosis. When all the previous illnesses are ruled out and a bone biopsy turns up negative for any known tumors, bacteria, or fungus, CRMO is usually diagnosed.

\section{ACKNOWLEDGEMENT}

Authors would like to acknowledge to the patient who permitted to get it published.

\section{AUTHOR'S CONTRIBUTION}

SG- wrote the entire article, did all the literature review and did all the edits. All Authors read and approved the article.

SOURCE OF SUPPORT: National Academy of Medical Sciences, Kathmandu

CONFLICT OF INTEREST: There's no conflict of interest regarding the publication of this paper. It hasn't been presented till date in any forms.

\section{REFERENCES}

1. Shanti E, Ferguson HI. Chronic recurrent multifocal osteomyelitis: a concise review and genetic update. Clinical Orthopaedics and Related Res 2007; 462:11-9.

2. Majeed H, Kalaawi M, Mohanty D, Teebi A, Tunjekar M, Algharbawy F, Majeed S, Algazzar A. Congenital dyserythropoietic anemia and chronic recurrent multifocal osteomylitis in three related children and the association with Sweet syndrome in two siblings. The Journal of Pediat 1989; 115 (5, Part 1):730-4.

3. Mosawi A, Saad A Maghsoodi I Shanti E. A splice site mutation confirms the role of LPIN2 in Majeed syndrome. Arthritis \& Rheumat 2007;56(3):960-4.
4. Brown, Robert, Wilkinson, Timothy. Chronic recurrent multifocal osteomylitis. Radiol 1988; 166(2):493-6.

5. Chiu CK, Singh VA. Chronic recurrent multifocal osteomyelitis of the first metatarsal bone: a case report. Journal of Orthopaedic Surg 2009;17 (1):119-22.

6. Gallagher KT, Roberts RL, MacFarlane JA, Stiehm ER. Treatment of chronic recurrent multifocal osteomyelitis (CRMO) with interferon-gamma. J Pediatr 1997; 131(3):470-2.

\section{Correspondence to:}

Dr. Samaj Gautam

Consultant orthopedic Surgeon

Department of Orthopedics and Trauma Surgery

National Academy of Medical Sciences

Email: samajgautam@gmail.com 